\title{
Boltzmann Collision Kernels and Velocity Saturation in Semiconductors
}

\author{
Christoph Dalitz \\ Universität Bielefeld, Theoretische Physik \\ Universitätsstr. 25, D-33615 Bielefeld
}

\begin{abstract}
For different models of the electron-phonon interaction, the asymptotic behaviour of the moments of the stationary homogeneous solution of the linear Boltzmann equation is determined in the limit of a high external field. For Hilbert-Schmidt kernels of a finite rank, a result recently proven for kernels of rank one is found generally valid; as a consequence velocity saturation is excluded for these collision models. For a class of singular collision kernels in contrast, velocity saturation is generally obtained.
\end{abstract}

\section{Introduction}

It is a well known fact that the drift velocity of electrons in a semiconductor approaches a finite value, if the applied field becomes very large. Already ShockLey, whose experiments with RYDER [1] first revealed saturation of the electron drift velocity in germanium, argued in an intuitive manner that optical phonon scattering should be responsible for this effect [2]. Meanwhile, changeover from acoustic to optic mode phonon emission in high electric fields has been examined experimentally in some detail [3].

For a mathematical explanation of velocity saturation, it is necessary to determine the high field behaviour of the first moment with respect to the momentum $p$ of the solution $f(p)$ of the stationary homogeneous Boltzmann equation [4]

$$
E \frac{\partial}{\partial p} f(p)=\mathcal{Q} f(p)
$$

For several specific models of the electron-phonon collision operator $\mathcal{Q}$, this behaviour has been determined by different authors [5], [6], [7]. Particularly ReIK and RISKEN have shown that in the presence of both optic and acoustic mode deformation potential scattering the electron drift velocity saturates [5]. Nevertheless the 
interesting question remains as to which general classes of collision models actually do or do not lead to velocity saturation.

In this article we answer this question for two classes of collision models within the framework of a parabolic band approximation and the assumption of a nondegenerate electron gas, which means that the electron concentration be not too high [8]. The main results are given in form of theorems at the end of section three and five.

In section three and four, we examine regular kernels: if their rank is finite, the high field behaviour of the moments is given by a law which the author has proven recently for "relaxation time approximations" (kernels of rank one) [7]. As a consequence, velocity saturation is excluded for these kernels. This result seems to remain valid for kernels of infinite rank as well, as we point out in section three.

In section five we shall see that the situation changes considerably, if the quantum nature of the lattice vibrations is taken into account. Under the assumption of a single phonon frequency ("EINSTEIN's model", which is a good approximation for the optical phonon mode [9]) and some isotropy hypothesis upon the scattering probability, it will turn out that the electron drift velocity saturates, regardless of the detailed functional form of the scattering probability.

\section{The collision operator}

If the electron concentration is low enough, so that interactions among the electrons themselves (via collisions or PAULI's exclusion principle) may be neglected, the electron phonon collision operator $\mathcal{Q}$ in (1) is linear and has the general form [4] (throughout this article we set the effective mass of the electrons equal unity, so that momentum $p$ and velocity are the same)

$$
\mathcal{Q} f(p)=M(p) \int d p^{\prime} K\left(p^{\prime}, p\right) f\left(p^{\prime}\right)-f(p) \int d p^{\prime} M\left(p^{\prime}\right) K\left(p, p^{\prime}\right)
$$

where $M(p):=(2 \pi \theta)^{-1 / 2} e^{-p^{2} / 2 \theta}$ is the Maxwellian at temperature $\theta$ of the semiconductor lattice and the collision kernel $K\left(p, p^{\prime}\right)$ is a symmetric and positive distribution. The second integral in (2), which gives the total scattering rate, is usually called collision frequency $\nu$

$$
\nu(p):=\int d p^{\prime} M\left(p^{\prime}\right) K\left(p, p^{\prime}\right)
$$

The collision kernel $K\left(p, p^{\prime}\right)$ strongly depends on the model for the electron-lattice interaction. We can roughly divide these models into two classes:

The first class are classical models, which allow an arbitrary amount of energy exchange between electrons and lattice; these models lead to continuous collision kernels or (under the not very restrictive assumption that $K$ be squareintegrable 
with weight $M)$ Hilbert-Schmidt kernels. Nearly all existence and uniqueness results for the linear Boltzmann equation refer to collision kernels belonging to this class.

The second class are semiclassical models, which take into account the quantum nature of the lattice vibrations. These models lead to singular kernels, where energy conservation is represented by Dirac $\delta$-distributions in the collision kernel.

\section{Hilbert-Schmidt kernels of finite rank}

Let us begin our examinations with collision kernels that are measurable functions with a finite norm

$$
\|K\|_{M}^{2}:=\int d p \int d p^{\prime} M(p) M\left(p^{\prime}\right) K^{2}\left(p, p^{\prime}\right)<\infty
$$

Since $K$ is symmetric, the kernel $K^{\prime}\left(p, p^{\prime}\right)=\sqrt{M(p)} K\left(p, p^{\prime}\right) \sqrt{M\left(p^{\prime}\right)}$ is symmetric and squareintegrable. Hence it follows from standard Hilbert-Schmidt theory [10 that $K^{\prime}$ has an eigenfunction representation which converges in $L^{2}\left(\mathbb{R}^{6}\right)$. If we write this representation in terms of $K$, we find

$$
K\left(p, p^{\prime}\right)=\sum_{n=0}^{\infty} \lambda_{n} \varphi_{n}(p) \varphi_{n}\left(p^{\prime}\right)
$$

where the right hand side converges with respect to the norm (4). The $n$-th eigenfunction $\varphi_{n}$ is a solution of the eigenvalue equation with eigenvalue $\lambda_{n}$

$$
\int d p^{\prime} M\left(p^{\prime}\right) K\left(p, p^{\prime}\right) \varphi_{n}\left(p^{\prime}\right)=\lambda_{n} \varphi_{n}(p)
$$

The eigenfunctions $\left\{\varphi_{n}\right\}$ form an orthonormal system with respect to the $L^{2}$-scalarproduct with weight $M$ :

$$
\int d p \varphi_{n}(p) \varphi_{m}(p) M(p)=\delta_{n m}
$$

Now let us assume that the eigenvalue equation (6) has a solution only for a finite number of eigenvalues $\left\{\lambda_{n}\right\}_{n=1}^{n_{\max }}$, which means that the kernel $K\left(p, p^{\prime}\right)$ is of finite rank. In this case the eigenfunction representation (5) takes the form

$$
K\left(p, p^{\prime}\right)=\sum_{n=1}^{n_{\max }} \lambda_{n} \varphi_{n}(p) \varphi_{n}\left(p^{\prime}\right)
$$

For the special case $n_{\max }=1,(8)$ is identical with the relaxation time approximation discussed in [7]. We shall see that the stationary homogeneous Boltzmann equation can be readily integrated for any finite $n_{\max }$ and the resulting expression for the moments allows an asymptotic evaluation for large fields $E$. 


\subsection{Solution of the Boltzmann equation}

With the collision kernel (8), the homogeneous stationary Boltzmann equation takes the form

$$
\left(E \partial_{p}+\sum_{n=1}^{n_{\max }} \mu_{n} \varphi_{n}(p)\right) f(p)=M(p) \sum_{n=1}^{n_{\max }} a_{n} \varphi_{n}(p)
$$

where we have introduced the abbreviations

$$
\mu_{n}:=\lambda_{n} \int_{-\infty}^{\infty} d p^{\prime} M\left(p^{\prime}\right) \varphi_{n}\left(p^{\prime}\right) \quad \text { and } \quad a_{n}:=\lambda_{n} \int_{-\infty}^{\infty} d p^{\prime} f\left(p^{\prime}\right) \varphi_{n}\left(p^{\prime}\right)
$$

From its definition (3) we see that the collision frequency in this model is given by $\nu(p)=\sum \mu_{n} \varphi_{n}(p)$. The solution of (9) vanishing at $p= \pm \infty$ is

$$
f(p)=\frac{1}{E} e^{-N(p) / E} \sum_{n=1}^{n_{\max }} a_{n} \int_{-\infty}^{p} d p^{\prime} \varphi_{n}\left(p^{\prime}\right) M\left(p^{\prime}\right) e^{N\left(p^{\prime}\right) / E}
$$

$N(p)$ (read "capital $\nu$ ") denotes the integral over the collision frequency

$$
\text { where } N(p):=\int_{0}^{p} d q \nu(q)=\int_{0}^{p} d q \sum_{n=1}^{n_{\max }} \mu_{n} \varphi_{n}(q)
$$

The coefficients $a_{n}$ must be determined selfconsistently by insertion of the solution (11) into the definition of the coefficients (10). This leads to the system of linear equations

$$
\sum_{n=1}^{n_{m a x}}\left(\mathrm{~A}_{m n}-\delta_{m n}\right) a_{n}=0
$$

where $\delta_{m n}$ denotes Kronecker's symbol and the matrix $\mathrm{A}_{m n}$ is given by

$$
\mathrm{A}_{m n}:=\frac{1}{E} \int_{-\infty}^{\infty} d p e^{-N(p) / E} \lambda_{m} \varphi_{m}(p) \int_{-\infty}^{p} d p^{\prime} \varphi_{n}\left(p^{\prime}\right) M\left(p^{\prime}\right) e^{N\left(p^{\prime}\right) / E}
$$

Partial integration in the $p$-integral using $N^{\prime}(p)=\sum \mu_{k} \varphi_{k}(p)$ reveals that

$$
\sum_{k=1}^{n_{\max }} \frac{\mu_{k}}{\lambda_{k}}\left(\mathrm{~A}_{k n}-\delta_{k n}\right)=0
$$

so that the determinant of the matrix $(\mathrm{A}-1)$ vanishes. Hence the homogeneous system (13) indeed has a nontrivial solution and the stationary homogeneous Boltzmann equation is solved by (11). 


\subsection{Asymptotic evaluation of the moments}

After changing the order of integrations we obtain for the $n$-th moment of $f$ given by (11) (remember that the solutions $a_{k}$ of (13) depend on $E$ )

$$
\begin{aligned}
\left\langle p^{n}\right\rangle(E) & =\frac{\int_{-\infty}^{\infty} d p p^{n} f}{\int_{-\infty}^{\infty} d p^{\prime} f}=\frac{\sum_{k=1}^{n_{\max }} a_{k}(E) I_{k}^{n}(E)}{\sum_{k=1}^{n_{\max }} a_{k}(E) I_{k}^{0}(E)} \quad \text { with } \\
I_{k}^{n}(E) & :=\int_{-\infty}^{\infty} d p e^{-p^{2} / 2 \theta} \varphi_{k}(p) e^{N(p) / E} \int_{p}^{\infty} d q q^{n} e^{-N(q) / E}
\end{aligned}
$$

If we make the substitutions $t=N(q)$ and $s=N(p)$, we can interpret the double integrals in (14) as Laplace transforms with the Laplace variable $\varepsilon:=1 / E$ (we denote the inverse function of $N$ by $\left.N^{-1}\right)$ :

$$
\begin{aligned}
I_{k}^{n}(E) & =\int_{-\infty}^{\infty} d s \frac{\varphi_{k}\left(N^{-1}(s)\right)}{\nu\left(N^{-1}(s)\right)} e^{-\left(N^{-1}(s)\right)^{2} / 2 \theta} \int_{s}^{\infty} d t \frac{\left(N^{-1}(t)\right)^{n}}{\nu\left(N^{-1}(t)\right)} e^{-(t-s) \varepsilon} \\
& =\int_{0}^{\infty} d t e^{-t \varepsilon} \int_{-\infty}^{\infty} d s \frac{\varphi_{k}\left(N^{-1}(s)\right)}{\nu\left(N^{-1}(s)\right)} \frac{\left(N^{-1}(t+s)\right)^{n}}{\nu\left(N^{-1}(t+s)\right)} e^{-\left(N^{-1}(s)\right)^{2} / 2 \theta}
\end{aligned}
$$

According to the Abelian theorems on Laplace transforms [11, the asymptotic behaviour of the Laplace transform (15) for small $\varepsilon$ is determined by the asymptotic behaviour of the inner integral for large $t$. Under the assumption that $N^{-1}(t)$ behaves asymptotically as given in (16) (see theorem 1 below), the asymptotic behaviour of the $s$-integral for large $t$ can be determined in complete analogy to section 4 of reference [7]. The result is

$$
s \text {-integral in }(15) \stackrel{t \rightarrow \infty}{\sim} \frac{\left(N^{-1}(t)\right)^{n}}{\nu\left(N^{-1}(t)\right)} \underbrace{\int_{-\infty}^{\infty} d s \frac{\varphi_{k}\left(N^{-1}(s)\right)}{\nu\left(N^{-1}(s)\right)} e^{-\left(N^{-1}(s)\right)^{2} / 2 \theta}}_{=\mu_{k} / \lambda_{k}}
$$

For small values of $\varepsilon=1 / E$, we may replace the $s$-integral in (15) by this asymptotic expression for large $t$. If we resubstitute $t=N(p)$ and insert the result into (14), we obtain

$$
\left\langle p^{n}\right\rangle(E) \stackrel{E \rightarrow \infty}{\sim} \frac{\sum_{k=1}^{n_{\max }} a_{k}(E) \frac{\mu_{k}}{\lambda_{k}} \int_{0}^{\infty} d p p^{n} e^{-N(p) / E}}{\sum_{k=1}^{n_{\max }} a_{k}(E) \frac{\mu_{k}}{\lambda_{k}} \int_{0}^{\infty} d p e^{-N(p) / E}}=\frac{\int_{0}^{\infty} d p p^{n} e^{-N(p) / E}}{\int_{0}^{\infty} d p e^{-N(p) / E}}
$$

This is exactly the same expression that was obtained in [7] for kernels of rank one in the zero temperature limit. Consequently we obtain the same high field behaviour of the moments (for details of the derivation see [7]): 
Theorem 1: The collision kernel be of the form (8) and the inverse function $N^{-1}$ of the integral over the collision frequency $N(p)=\int_{0}^{p} \nu(q) d q$ have the asymptotic behaviour

$$
N^{-1}(t) \sim A t^{\alpha} L(t) \quad \text { for } \quad t \rightarrow \infty
$$

where $\alpha>-1$ and $L$ be a continuous function with the property $\lim _{t \rightarrow \infty} L(\tau t) / L(t)=$ 1 for every $\tau>0$.

Then the $n$-th moment of the solution of the stationary homogeneous Boltzmann equation behaves for $E \rightarrow \infty$ like

$$
\left\langle p^{n}\right\rangle \sim \frac{A^{n}}{n+1} \frac{\Gamma(n \alpha+\alpha+1)}{\Gamma(\alpha+1)} E^{n \alpha} L^{n}(E) \sim \text { const } \cdot\left(N^{-1}(E)\right)^{n}
$$

In particular the first moment or the drift velocity behaves as $N^{-1}(E)$ for large $E$. Because of $N(\infty)=\infty$, which is a necessary condition for the existence of a stationary homogeneous solution of the Boltzmann equation [12], we conclude that velocity saturation is excluded for Hilbert Schmidt kernels of finite rank.

\section{A conjecture}

Since we have seen that theorem 1 on the high field behaviour of the moments is valid for Hilbert-Schmidt kernels of any finite rank, it is reasonable to assume that our theorem holds for arbitrary Hilbert-Schmidt kernels as well. This conjecture however is very difficult to prove; a major problem is that the eigenfunctions are not positive for a rank greater than one, so that their sum (5) is not necessarily positive if it is truncated at finite $n$. Maybe a proof is possible, if the kernel $K\left(p, p^{\prime}\right)$ is a continuous function of $p$ and $p^{\prime}$, for then the sum (5) converges even uniformly. Nevertheless the problem mentioned above still remains.

As a reinforcement of our conjecture, let us consider the kernel of infinite rank $K\left(p, p^{\prime}\right)=\rho\left|p-p^{\prime}\right|$, for which GeRvois and Piasecki determined the large field behaviour of the first moment in [6]. They obtained

$$
\langle p\rangle(E) \sim \sqrt{2 E / \rho \pi} \text { for } E \rightarrow \infty
$$

which is independent of the temperature $\theta$. In this model, the collision frequency reads

$$
\nu(p)=2 \rho \theta M(p)+\rho|p|\left(1-2 \int_{|p|}^{\infty} M\left(p^{\prime}\right) d p^{\prime}\right)
$$

Hence, the integral over the collision frequency behaves as $N(p) \sim \rho p^{2} / 2$ for large $p$ and its inverse function as $N^{-1}(t) \sim \sqrt{2 t / \rho}$ for large $t$. Naive application of theorem 1 leads to

$$
\langle p\rangle(E) \stackrel{E \rightarrow \infty}{\sim} \sqrt{2 / \rho} \frac{\Gamma(1+1)}{2 \Gamma(0.5+1)} \sqrt{E}=\sqrt{2 E / \rho \pi}
$$


which is identical with Gervois' and PiASECKI's result (18)!

\section{Optical phonon scattering}

Now let us consider collision models that take into account the quantum nature of the lattice vibrations. Under the assumption of a single phonon energy $\omega$ (this assumption holds for the optical mode), the general form of the collision kernel reads 13]

$$
\begin{aligned}
M(p) K\left(p^{\prime}, p\right)= & \beta\left(p^{\prime}, p\right)\left\{\left(N_{o}+1\right) \delta\left(p^{2} / 2-p^{\prime 2} / 2+\omega\right)\right. \\
& \left.+N_{o} \delta\left(p^{2} / 2-p^{\prime 2} / 2-\omega\right)\right\}
\end{aligned}
$$

where $N_{o}=\left(e^{\omega / \theta}-1\right)^{-1}$ is the occupation number of the optical phonons with energy $\omega$ and the scattering cross section $\beta\left(p^{\prime}, p\right)$ is a symmetric and positive function. The first term in the curly braces describes phonon emission, the second phonon absorption during the scattering process; the Dirac $\delta$-distributions assure energy conservation.

Under the isotropy assumption $\beta=\beta\left(\left|p^{\prime}\right|,|p|\right)$, we shall see that an asymptotic solution of the Boltzmann equation is possible for high energy electrons with $p^{2} \gg 2 \omega$, provided the electric field is sufficiently high $E \gg \omega$. The asymptotic knowledge of the electron distribution will be sufficient for the determination of the high field behaviour of the moments.

Our replacement of difference operators by differential operators, which is the essential step in our procedure, is not new. The first who did so were Yamashita and Watanabe [14]. However, they obtained erroneous results for the electron distribution and the drift velocity, which were corrected by REIK and RISKEN [5]. HÄNSCH and SCHWERIN have applied this method to inhomogeneous situations as well [15]. In all three references, a combination of acoustic and optical phonon scattering is considered and the scattering cross section $\beta$ is taken to be constant.

\subsection{The stationary homogeneous Boltzmann equation}

Under the isotropy assumption $\beta\left(p^{\prime}, p\right)=\beta\left(\left|p^{\prime}\right|,|p|\right)$, the one dimensional stationary homogeneous Boltzmann equation with the collision kernel (19) reads

$$
\begin{aligned}
& \left\{E \partial_{p}+\left(N_{o}+1\right) \Theta\left(p^{2}-2 \omega\right) \beta^{-}(p)+N_{o} \beta^{+}(p)\right\} f(p) \\
& \quad=\left(N_{o}+1\right) \beta^{+}(p) \frac{1}{2}\left\{f\left(-\sqrt{p^{2}+2 \omega}\right)+f\left(+\sqrt{p^{2}+2 \omega}\right)\right\} \\
& \quad+N_{o} \Theta\left(p^{2}-2 \omega\right) \beta^{-}(p) \frac{1}{2}\left\{f\left(-\sqrt{p^{2}-2 \omega}\right)+f\left(+\sqrt{p^{2}-2 \omega}\right)\right\}
\end{aligned}
$$


where $\Theta$ denotes Heaviside's step function, and the abbreviation $\beta^{ \pm}$means

$$
\beta^{ \pm}(p):=2 \beta\left(\sqrt{p^{2} \pm 2 \omega}, p\right) / \sqrt{p^{2} \pm 2 \omega}
$$

Now let us split $f(p)=f_{o}(p)+f_{e}(p)$ into its odd and even part $f_{o}(p):=(f(p)-$ $f(-p)) / 2$ and $f_{e}(p):=(f(p)+f(-p)) / 2$. Then we may write (20) as two coupled equations for $f_{o}$ and $f_{e}$ for $p>0$. With the substitution $q:=p^{2} / 2$ and the notations

$$
\begin{aligned}
& F_{e}(q):=f_{o}(\sqrt{2 q})=f_{e}(p)=\frac{1}{2}(f(p) \mp f(-p)) \quad \text { and } \\
& B(q):=\beta(\sqrt{2(q-\omega)}, \sqrt{2 q}) / \sqrt{q(q-\omega)}
\end{aligned}
$$

we obtain the coupled system of equations for $F_{o}$ (remember that $\beta^{ \pm}$are even functions of $p$ and that $\beta\left(p^{\prime}, p\right)$ is symmetric with respect to exchange of $p$ and $\left.p^{\prime}\right)$

$$
\begin{aligned}
E \partial_{q} F_{o}(q)= & \left(N_{o}+1\right) B(q+\omega) F_{e}(q+\omega)+N_{o} \Theta(q-\omega) B(q) F_{e}(q-\omega) \\
& -\left[\left(N_{o}+1\right) \Theta(q-\omega) B(q)+N_{o} B(q+\omega)\right] F_{e}(q) \\
E \partial_{q} F_{e}(q)= & -\left[\left(N_{o}+1\right) \Theta(q-\omega) B(q)+N_{o} B(q+\omega)\right] F_{o}(q)
\end{aligned}
$$

Using (21), we may express the moments of the electron distribution in terms of the functions $F_{o}$

$$
\left\langle p^{n}\right\rangle(E)=\frac{\int_{-\infty}^{\infty} d p p^{n} f}{\int_{-\infty}^{\infty} d p f}= \begin{cases}\frac{\int_{0}^{\infty} d q(2 q)^{\frac{n-1}{2}} F_{o}(q)}{\int_{0}^{\infty} d q F_{e}(q) / \sqrt{2 q}} & \text { for odd } n \\ \frac{\int_{0}^{\infty} d q(2 q)^{\frac{n-1}{2}} F_{e}(q)}{\int_{0}^{\infty} d q F_{e}(q) / \sqrt{2 q}} & \text { for even } n\end{cases}
$$

\subsection{Asymptotic solution for fast electrons}

For sufficiently high electric fields, an asymptotic solution of (23) in the region $p^{2} \gg$ $2 \omega$ is possible via replacement of the difference operators by differential operators. For this purpose we switch to the new varible $x:=q / E$ and denote the resulting new functions with hats:

$$
\hat{F}_{e}(x):=F_{e}(E x)=F_{e}(q) \quad \text { and } \quad \hat{B}(x):=B(E x)=B(q)
$$

After a rearrangement of terms, the system (23) reads for $x>\epsilon:=\omega / E$

$$
\begin{aligned}
\partial_{x} \hat{F}_{o}(x)= & \left(N_{o}+1\right)\left\{\hat{B}(x+\epsilon) \hat{F}_{e}(x+\epsilon)-\hat{B}(x) \hat{F}_{e}(x)\right\} \\
& -N_{o}\left\{\hat{B}(x+\epsilon) \hat{F}_{e}(x)-\hat{B}(x) \hat{F}_{e}(x-\epsilon)\right\} \\
\partial_{x} \hat{F}_{e}(x)= & -\left\{\left(N_{o}+1\right) \hat{B}(x)+N_{o} \hat{B}(x+\epsilon)\right\} \hat{F}_{o}(x)
\end{aligned}
$$


For small $\epsilon \ll 1$ and large $x \gg \epsilon$ (which means $p^{2} \gg 2 \omega$ ), the difference operators on the right hand side can be replaced by differential operators. If we make a Taylor expansion of $\hat{F}_{e}(x \pm \epsilon)$ and $\hat{B}(x+\epsilon)$ around $x$ and keep only terms up to first order in $\epsilon$, we obtain

$$
\begin{aligned}
& \partial_{x} \hat{F}_{o}(x) \approx \epsilon \partial_{x}\left(\hat{B}(x) \hat{F}_{e}(x)\right) \\
& \partial_{x} \hat{F}_{e}(x) \approx-\left\{\left(2 N_{o}+1\right) \hat{B}(x)+\epsilon N_{o} \partial_{x} \hat{B}(x)\right\} \hat{F}_{o}(x)
\end{aligned}
$$

Taking into account that $\hat{F}_{o}(\infty)=0$, we may integrate (26a) from $x$ to infinity and insert the result into (26b). Again we keep only terms up to first order in $\epsilon$.

$$
\begin{aligned}
\hat{F}_{o}(x) & \approx \epsilon \hat{B}(x) \hat{F}_{e}(x) \\
\partial_{x} \hat{F}_{e}(x) & \approx-\epsilon\left(2 N_{o}+1\right) \hat{B}^{2}(x) \hat{F}_{e}(x)
\end{aligned}
$$

Equation (27b) easily can be integrated with the boundary condition $\hat{F}_{e}(\infty)=0$. Thus we obtain an asymptotic solution of (25) for $x \gg \epsilon$ under the assumption that $\epsilon=\omega / E$ be small. We may also take into account the region where $x \gg \epsilon$ does not hold by introduction of two functions $\varrho_{e}(t)$ with the property $\lim _{t \rightarrow \infty} \varrho_{e}(t)=1$. Then the factors $\varrho_{o}(x / \epsilon)$ describe transition to the asymptotic domain $x \gg \epsilon$ and the solution globally valid in $x$ reads

$$
\begin{aligned}
& \hat{F}_{e}(x) \stackrel{\epsilon \ll 1}{\approx} C \varrho_{e}(x / \epsilon) \exp \left(\epsilon\left(2 N_{o}+1\right) \int_{0}^{x} d y \hat{B}^{2}(y)\right) \\
& \hat{F}_{o}(x) \stackrel{\epsilon \ll 1}{\approx} C \varrho_{o}(x / \epsilon) \epsilon \hat{B}(x) \exp \left(\epsilon\left(2 N_{o}+1\right) \int_{0}^{x} d y \hat{B}^{2}(y)\right)
\end{aligned}
$$

with some arbitrary constant $C>0$.

\subsection{High field behaviour of the moments}

If we insert the high field solution (28) into the expression for the moments (24), we find after the substitutions $q=E x$ and $q^{\prime}=E y$ (remember that $\epsilon=\omega / E$ )

$$
\begin{aligned}
& \left\langle p^{n}\right\rangle(E) \stackrel{E \gg \omega}{{ }^{\omega}} I_{n}(E) / I_{0}(E) \quad \text { with } \\
& I_{n}(E)= \begin{cases}\frac{\omega}{E} \int_{0}^{\infty} d q(2 q)^{\frac{n-1}{2}} \varrho_{o}\left(\frac{q}{\omega}\right) B(q) \exp \left(-\frac{\omega\left(2 N_{o}+1\right) A(q)}{E^{2}}\right) & \text { for odd } n \\
\int_{0}^{\infty} d q(2 q)^{\frac{n-1}{2}} \varrho_{e}\left(\frac{q}{\omega}\right) \exp \left(-\frac{\omega\left(2 N_{o}+1\right) A(q)}{E^{2}}\right) & \text { for even } n\end{cases}
\end{aligned}
$$

where we have introduced the abbreviation

$$
A(q):=\int_{0}^{q} d q^{\prime} B^{2}\left(q^{\prime}\right)
$$

As $E$ goes to infinity, the integrals in the numerator and denominator of (29) diverge, whilst the integrals remain finite if the upper limit is replaced by a finite value. 
Hence their behaviour for large fields is determined by the asymptotic behaviour of the integrands for large $q$ and consequently our knowledge of the asymptotic form of the electron distribution for $p^{2} \gg 2 \omega$ will suffice for the determination of the high field moments.

For convenience, let us assume that $A(\infty)=\infty$. Then we can procede as in section 3 by writing the integrals in (30) as Laplace transforms, which we can evaluate with the Abelian theorem again. To this purpose we make the substitution $t=A(q)$ and obtain for large values of $E$ (the inverse function of $A$ is denoted by $A^{-1}$ )

$$
I_{n}(E) \sim \begin{cases}\frac{\omega}{E} \int_{0}^{\infty} d t \frac{\left(2 A^{-1}(t)\right)^{\frac{n-1}{2}}}{B\left(A^{-1}(t)\right)} \exp \left(-\frac{\omega\left(2 N_{o}+1\right) t}{E^{2}}\right) & \text { for odd } n \\ \int_{0}^{\infty} d t \frac{\left(2 A^{-1}(t)\right)^{\frac{n-1}{2}}}{B^{2}\left(A^{-1}(t)\right)} \exp \left(-\frac{\omega\left(2 N_{o}+1\right) t}{E^{2}}\right) & \text { for even } n\end{cases}
$$

Under the assumption that $B(q)$ asymptotically grows like a power for large values of $q$, the asymptotic large field behaviour of the integrals in (32) is easily evaluated with the aid of the Abelian theorem on Laplace transforms ([1] part I, chapter 13 $\S 1$ theorem 7 ). The elementary calculation leads to

Theorem 2: The collision operator be of the form (19) and the scattering cross section $\beta$ satisfy the isotropy property $\beta\left(p^{\prime}, p\right)=\beta\left(\left|p^{\prime}\right|,|p|\right)$ and be such that for $q \rightarrow \infty$

$$
B(q)=\beta(\sqrt{2(q-\omega)}, \sqrt{2 q}) / \sqrt{q(q-\omega)} \sim a \cdot q^{\alpha}
$$

with some constants $a>0$ and $\alpha \geq-1 / 2$.

Then the $n$-th moment of the solution of the stationary homogeneous Boltzmann equation behaves for $E \rightarrow \infty$ like

$$
\left\langle p^{n}\right\rangle(E) \sim \begin{cases}2^{\frac{n}{2}} a \omega \frac{\Gamma\left(\frac{n+1+2 \alpha}{4 \alpha+2}\right)}{\Gamma\left(\frac{1}{4 \alpha+2}\right)}\left(\frac{2 \alpha+1}{a^{2} \omega\left(2 N_{o}+1\right)}\right)^{\frac{n+2 \alpha}{4 \alpha+2}} E^{\frac{n-1}{2 \alpha+1}} & \text { for odd } n \\ 2^{\frac{n}{2}} \frac{\Gamma\left(\frac{n+1}{4 \alpha+2}\right)}{\Gamma\left(\frac{1}{4 \alpha+2}\right)}\left(\frac{2 \alpha+1}{a^{2} \omega\left(2 N_{o}+1\right)}\right)^{\frac{n}{4 \alpha+2}} E^{\frac{n}{2 \alpha+1}} & \text { for even } n\end{cases}
$$

Particularly, for the first moment we find

$$
\langle p\rangle(E) \sim \frac{\Gamma\left(\frac{\alpha+1}{2 \alpha+1}\right)}{\Gamma\left(\frac{1}{4 \alpha+2}\right)} \sqrt{\frac{(4 \alpha+2) \omega}{2 N_{o}+1}}
$$

which is a finite value. Consequently the drift velocity saturates if the electrons interact with optical phonons! 


\subsection{An example}

For the particular choice $\beta\left(p, p^{\prime}\right)=C|p| \cdot\left|p^{\prime}\right|$ proposed by MAHAN [16, the function $B(q)$ defined by (22) is simply a constant, so that the stationary homogeneous Boltzmann equation can be solved exactly in the zero temperature limit $N_{o}=0$ [17]. The resulting drift velocity is

$$
\langle p\rangle(E)=\frac{\omega / z E}{\sqrt{2 \omega}+e^{z \omega} \sqrt{\pi / 2 z} \operatorname{erfc}(\sqrt{z \omega})}
$$

where $z$ is the positive real root of the transcendental equation $(z E)^{2}=1-e^{-z \omega}$. For large $E$, this solution is approximately $z(E) \approx \omega / E^{2}$ so that the current approaches the finite value $j_{\text {sat }}=\sqrt{2 \omega / \pi}$ as $E$ goes to infinity. This result is in agreement with (35) for $N_{o}=0$ and $\alpha=0$.

In this model the asymptotic formula for the drift velocity is easily improved by keeping terms up to second order in (25). If we do so, we find for the first moment

$$
\langle p\rangle(E) \sim \sqrt{\frac{2 \omega}{\pi\left(1+\omega^{2} / 2 E^{2}\right)}}
$$

A comparison of our asymptotic formula (37) with the exact value (36) is given in the figure. The agreement for large values of the electric field $E$ is clearly visible. (The figure is omitted in the cond_mat-preprint).

\section{Summary and conclusions}

In this article we have seen that the experimenatlly observed saturation of the electron drift velocity in semiconductors cannot be obtained with regular collision kernels of finite rank. This result seems to remain valid even for infinite rank, though we could not present a rigorous proof of this conjecture. An extension of our argumentation to three dimensions is straightforward and can be performed in complete analogy to the case of rank one [7].

In his investigation of the null space of the Boltzmann collision operator [18], MAJORANA has already observed that singular collision kernels have mathematical properties rather different from regular collision kernels. In this article we have seen that also velocity saturation is a feature restricted to singular kernels.

Under the assumption of isotropic optical phonon scattering we were able to derive velocity saturation, regardless of the detailed form of the scattering cross section. However two interesting questions remain, which are worth further investigation. First, is there a generalization of our proof to three dimensions beyond the framework of a two term Legendre approximation? The latter approximation, used in [14] and [5], is an effective one dimensional approximation, which is equivalent to our one dimensional model. Second, does our result remain unchanged if the scattering is anisotropic? 


\section{Acknowledgement}

The author is grateful to the "Studienstiftung des deutschen Volkes" for financial support of this work.

\section{References}

[1] E. J. Ryder, W. Shockley, Phys. Rev. $\underline{81}$ (1951) 139

[2] W. Shockley, Bell Syst. Techn. J. $\underline{30}$ (1951) 990

[3] P. Hawker, A. J. Kent, O. H. Hughes, L. J. Challis, Semicond. Sci. Technol. 7 (1992) B29

[4] P. A. Markowich, C. A. Ringhofer, C. Schmeiser: Semiconductor Equations. Springer, Wien, New York (1990)

[5] H. G. Reik, H. Risken, Phys. Rev. $\underline{124}$ (1961) 777

[6] A. Gervois, J. Piasecki, J. Stat. Phys. $\underline{42}$ (1986) 1091

[7] C. Dalitz, Physica A $\underline{232}$ (1996) 445

[8] F. Fuchs, Transp. Th. Stat. Phys. 25 (1996) 151

[9] R. Becker: Theorie der Wärme. 3. Auflage, Springer, Berlin (1985)

[10] S. Fenyö, H. W. Stolle: Theorie und Praxis der linearen Integralgleichungen. Birkhäuser, Basel (1982)

[11] G. Doetsch: Handbuch der Laplacetransformation. Birkhäuser, Basel (1970)

[12] F. Poupaud, Z. angew. Math. Mech. $\underline{72}$ (1992) 359

[13] B. Niclot, P. Degond, F. Poupaud, J. Comp. Phys. $\underline{78}$ (1988) 313

[14] J. Yamashita, M. Watanabe, Progr. Theor. Phys. 12 (1954) 443

[15] W. Hänsch, A. v. Schwerin, J. Appl. Phys. $\underline{66}$ (1989) 1435

[16] G. D. Mahan, J. Appl. Phys. $\underline{58}$ (1985) 2242

[17] C. Dalitz, Physica A $\underline{203}$ (1994) 125

[18] A. Majorana, Transp. Th. Stat. Phys. 20 (1991) 261 